\title{
What Ought a Fruitful Explicatum to be?
}

\author{
Mark Pinder ${ }^{1}$ (D)
}

Received: 9 April 2019 / Accepted: 13 January 2020 / Published online: 15 February 2020

(C) The Author(s) 2020

\begin{abstract}
Many concepts are inadequate for serious inquiry, so theorists often seek to engineer new concepts. The method of explication, which involves replacing concepts with more fruitful alternatives, is a model of this process. In this paper, I develop an account of fruitfulness, the Relevant-Goals Account of Fruitfulness. The account is in the spirit of extant proposals, but develops and extends them in important ways. In particular, while it applies to explications in general, the account allows us to derive substantive details for specific explications. This is achieved through a supplementary view, Contextualism, that specifies which theoretical goals are relevant in any given case. I close by discussing how the Relevant-Goals Account fits into the dynamic nature of inquiry.
\end{abstract}

\section{Introduction}

Many concepts are inadequate for serious inquiry. They may be insufficiently precise, they may cut across theoretically important distinctions, they may be politically charged, or they may be deficient in some other way. To deal with this, theorists often seek to refine their concepts. This is called conceptual engineering.

We can think of the method of explication as a model of conceptual engineering in inquiry. ${ }^{1}$ The method involves replacing one concept, the explicandum, with a newly constructed counterpart, the explicatum, for some or other theoretical purpose. Explicanda can be folk concepts or concepts from an earlier stage of inquiry; and explicata can (but need not) be expressed using the same words as corresponding explicanda. An application of the method is an explication. Explications can be performed by anyone or anything capable of undertaking inquiry, including individual theorists and institutions.

\footnotetext{
1 See, e.g., Carnap (1947, 1962, 1963) and Quine (1960). For recent discussions, see e.g. Bielik (2018), Brun (2016, 2017), Carus (2007), Dutilh Novaes and Reck (2017), Justus (2012), Kitcher (2008), Maher (2007), Pinder (2017a, b), Schupbach (2017) and Shepherd and Justus (2015).

Mark Pinder

Mark.Pinder@open.ac.uk

1 Department of Philosophy, The Open University, Walton Hall, Milton Keynes MK7 6AA, UK
} 
Following Carnap, it is standard to characterise explicata as being subject to four desiderata. In the ideal case, an explicatum is: similar to the explicandum (perhaps in the sense of having a similar function or playing a similar theoretical role); exact (in the sense of having precise rules of use or a precise definition); fruitful; and simple. It is not required that an explicatum satisfy these desiderata perfectly, ${ }^{2}$ only that the desiderata be satisfied to "a sufficient degree" (1962: 7). In general, an explication is successful when the explicatum is both: appropriately similar to the explicandum; and better on balance with respect to exactness, fruitfulness and simplicity.

There is a growing consensus that, in constructing an explicatum, fruitfulness is particularly important. ${ }^{3}$ Shepherd and Justus characterise fruitfulness as "the agenda" (2015: 388); and Dutilh Novaes and Reck write that "fruitfulness is ultimately the most significant requirement for an explication overall" (2017: 202). There are at least two ways to understand such claims. First, fruitfulness might be weighted more heavily than the other desiderata, so that a small increase in fruitfulness is preferable to a larger increase in (say) exactness. Or, second, fruitfulness might subsume other desiderata so that, in particular, exactness and simplicity are only desirable as a means to fruitfulness. ${ }^{4}$ Either way, on such a view, fruitfulness is the principal aim when undertaking the method of explication.

If right, we need a substantive account of fruitfulness. In this paper, I motivate and develop such an account: the Relevant-Goals Account of Fruitfulness. The account is offered within the spirit of recent discussions of fruitfulness, but refines and extends them in important ways. On the Relevant-Goals Account, fruitfulness is tied to the extent that an explication facilitates progress towards achieving relevant theoretical goals. This is intended as a general account but, importantly, it can be used to derive more substantive accounts for specific explications by specifying what the relevant theoretical goals are in each case. To this end, I develop a supplementary view, Contextualism, that specifies which theoretical goals are relevant in any given case. Finally, I discuss how the Relevant-Goals Account fits into the dynamic nature of inquiry.

Let me make several preliminary comments. First, as the focus herein is on fruitfulness, I put other desiderata to one side. Second, herein, I will not respond to general objections to the method of explication, such as Strawson's (1963) complaint that explication 'changes the subject'. 5 Third, I do not require an explication to involve formalisation: actual scientific and philosophical practice often involves the

\footnotetext{
${ }^{2}$ If explication is a model of conceptual refinement, perfect similarity is moreover undesirable. Cf. Dutilh Novaes and Reck's (2017) 'paradox of adequate formalization'.

3 Not everyone agrees. In particular, Schupbach (2017) develops 'Oppenheimian explication', a method designed to illuminate explicanda by maximising similarity to explicandum rather than fruitfulness. However, Schupbach accepts Carnap's understanding of fruitfulness which, I argue in Sect. 2, is too narrow. Once we accept a broader notion of fruitfulness, such as that developed herein, we can reconstrue Schupbach as seeking an explicatum that is fruitful for the specific purpose of illuminating the explicandum. See Pinder (2017b; Sect. 5.1).

4 This claim is more-or-less explicit in e.g. Dutilh Novaes and Reck (2017, pp. 199n7, 202), Shepherd and Justus (2015, p. 388) and Kitcher (2008, p. 119).

5 For responses, see e.g.: Brun (2016), Carnap (1963), Carus (2007), Dutilh Novaes and Reck (2017), Justus (2012), Kitcher (2008), Maher (2007), Pinder (2017b, 2019) and Schupbach (2017).
} 
construction of concepts that are informal or, at least, not fully formalised. ${ }^{6}$ Fourth, I take the method of explication to be normative, rather than descriptive, in the following sense: the method is a model of how theorists should engineer concepts, rather than how theorists in fact engineer concepts. I thus use "fruitfulness" as a term-of-art that denotes whatever theorists ought to aim for when engineering their concepts. Fifth, if we are to have any confidence in the method, we should nonetheless expect clear continuity with actual theoretical practice. For this reason, I focus the subsequent discussion around a historical case that fits the general structure of the method of explication. I call it the planet case. ${ }^{7}$

In the previous century, there was no accepted definition for the concept of planet. It was explicitly accepted that the concept-call it PLANET $_{\mathrm{OLD}}$ - had nine canonical instances, Mercury through to Pluto, and a number of canonical non-instances, such as the Sun, the Moon, asteroids, etc. However, in the early twenty first century, astronomers discovered several 'trans-Neptunian objects', such as Eris. These objects were similar in relevant respects both to Pluto and to asteroids, and thus seemed to be borderline cases of PLANET ${ }_{\mathrm{OLD}}$. As a result, in 2006, the International Astronomical Union (IAU) engineered a new concept, explicitly defined in terms of key properties of celestial objects, to replace PLANET ${ }_{\mathrm{OLD}}$ and facilitate a more principled classification of Pluto and other celestial objects.

Simplifying somewhat, the IAU considered two candidate explicata, PLANET ${ }_{\text {DRAFT }}$ and PLANET $\mathrm{NEW}_{\mathrm{N}}$, defined along the following lines. ${ }^{8}$

A celestial object $x$ falls under PLANET ${ }_{\text {DRAFT }}$ iff:

(a) $x$ orbits the sun; and

(b) $x$ is sufficiently large for its own gravity to have formed it into a sphere.

A celestial object $x$ falls under PLANET NEW $_{\text {iff: }}$

(a) $x$ orbits the sun;

(b) $x$ is sufficiently large for its own gravity to have formed it into a sphere; and

(c) $x$ has cleared its neighbourhood of debris.

Both Pluto and Eris fall under PLANET ${ }_{\text {DRAFT }}$; neither fall under PLANET ${ }_{\text {NEW }}$.

Following a vote, the IAU replaced PLANET ${ }_{\mathrm{OLD}}$ with PLANET $_{\mathrm{NEW}}$ : the institution and its members now use PLANET ${ }_{\mathrm{NEW}}$ for celestial taxonomisation, instead of using $\mathrm{PLANET}_{\mathrm{OLD}}$. As such, the IAU count as having explicated $\mathrm{PLANET}_{\mathrm{OLD}}$. A key question is whether the IAU made the right choice-whether PLANET ${ }_{\mathrm{NEW}}$ is more fruitful than PLANET $_{\text {DRAFT. }}$ To answer this question, we need an account of fruitfulness.

\footnotetext{
${ }^{6}$ Cf. Brun (2016: 1217f), Dutilh Novaes and Reck (2017: 673).

7 See Tyson (2009) for historical discussion.

8 See International Astronomical Union (2006a, b).
} 


\section{Previous Proposals}

Carnap characterises fruitfulness thus:

The explicatum is to be a fruitful concept, that is, useful for the formulation of many universal statements (empirical laws in the case of a nonlogical concept, logical theorems in the case of a logical concept). (1962: 7)

For example, Carnap writes that the nonlogical concept TEMPERATURE "has proved its great fruitfulness by the fact that it occurs in many important laws" (p. 14), such as the ideal gas law; and he hints that Frege's and Russell's set-theoretic definitions of ordinary arithmetical concepts (such as ZERO, ONE, ADDITION, etc.) are fruitful because, "[o]n the basis of this interpretation of the arithmetical terms, Peano's axioms [of arithmetic] become provable theorems in logic" (p. 17).

Carnap's proposal can be understood to consist of two independent claims ${ }^{9}$ :

(C1) A concept is fruitful insofar as it furthers the aims of empirical and logical inquiry.

(C2) The aim of empirical and logical inquiry is to formulate (and test, prove, etc.) universal statements (empirical laws and logical theorems). ${ }^{10}$

Now, the goal in the present paper is to find an account of fruitfulness that can serve as the aim for conceptual engineering in inquiry in general. However, for two reasons, Carnap's proposal is not, as it stands, suitable for this particular task. First, (C1) focuses only on empirical and logical inquiry. But, as illustrated by much of philosophy, inquiry need be neither empirical nor logical-some inquiry is, for example, conceptual or normative. Second, contrary to (C2), the aims of some areas of empirical inquiry are not best understood in terms of universal statements. As Kitcher writes, Carnap's proposal “doesn't suit parts of the physical sciences, and [is] deeply problematic for the biological, earth, and human sciences" (2008: 115). Such sciences do not trade in the kind of universal statements that are more familiar in, say, classical physics and mathematics.

Can we amend Carnap's account in some way? Kitcher proposes an amendment, intended to yield an account of fruitful concepts in science:

(K1) A concept is fruitful (qua science) insofar as it furthers the aims of science.

(K2) The aim of science is to provide "answers to significant questions, where the sources of significance are various, sometimes practical, sometimes in terms of the satisfaction of disinterested curiosity" (2008: 115).

\footnotetext{
9 See e.g. Kitcher (2008).

${ }^{10}$ As an anonymous reviewer notes, Carnap later explicitly denied that all scientific laws are universal: there are also statistical laws (1966: 3). This does not affect the criticism that follows; in general, the sciences are not best understood in terms of laws, whether universal or statistical. See e.g. Kitcher (2008: 119-122).
} 
As science is only one area of inquiry, this proposal as it stands is not a suitable account of the aim of conceptual engineering in inquiry in general. But the proposal is easily generalised:

(K1') A concept is fruitful insofar as it furthers the aims of inquiry.

(K2') The aim of inquiry is to provide answers to significant questions.

Now, this proposal has a twofold benefit. On the one hand, the idea of 'answering significant questions' is general enough to apply to any given field of inquiry: with enough care, most significant theoretical advances might be construed as answers to significant questions. But nonetheless, the proposal can be used to derive more substantive accounts of fruitfulness for specific fields of inquiry, by specifying what counts as a significant question in those fields. For example, in assessing an explicatum for the concept GENE, Kitcher highlights

four questions on which that explication might bear, ranging from issues of principally philosophical import to those that affect everyday biological research: (1) Can one reduce genetics to physics and chemistry [...]? (2) Is it possible to give a sense to the locution 'gene for $X^{\prime}$ [...] without lapsing into genetic determinism? (3) How can we discover the genes in a completely sequenced genome? (4) How does one distinguish genomes according to their relative complexity? (pp. 115-116)

These questions add clear, substantive detail to what counts as fruitfulness in this particular case. In general, then, the proposal promises to combine generality with substantive detail.

A less attractive aspect of the proposal-which applies mutatis mutandis to all those considered above - is its appeal to the aims of inquiry. There are least two concerns here. Firstly, it is unclear that inquiry as such has any aims at all; perhaps, instead, it is only theorists that have aims. ${ }^{11}$ There might, for example, be no nonarbitrary way to aggregate theorists' aims and practices to ground an aim for inquiry as such. ${ }^{12}$ Secondly, even if inquiry as such does have aims, it is unclear that we have easy epistemic access to what those aims are. It is certainly not obvious that inquiry, over and above the theorist, aims to answer significant questions; nor that it aims for truth, knowledge, empirical adequacy, or anything else.

Concerns about the aims of inquiry, while far from decisive, are easily circumvented: the appeal to such aims can be bypassed. Consider Dutilh Novaes and Reck's proposal that, when performing an explication, the "goal is to produce new

\footnotetext{
11 See, e.g., Rowbottom (2014).

12 As an anonymous referee points out, individual inquiries might have aims-to answer a particular question, to solve a particular problem, or something else. Plausibly, in such a case, the aim of the inquiry is grounded in the aims or practices of those undertaking the inquiry: if I aim to determine what 'fruitfulness' should be, then the aim of my inquiry is likewise to determine what 'fruitfulness' should be. This observation, however, does not help us to aggregate theorists' aims and practices to ground an aim for inquiry as such.
} 
knowledge about the phenomena to which the explicandum pertains" (2017: 206). This suggests:

(DNR) A concept is fruitful insofar as it facilitates the production of new knowledge about the phenomena to which it pertains.

In contrast to the previous proposals, (DNR) does not characterise fruitfulness in terms of the aims of inquiry. So we can accept (DNR), and be guided by it, without committing to the claim that inquiry as such has aims.

However, while (DNR) is an improvement upon (K1')-(K2') in this respect, it lacks the twofold benefit. It is perhaps the case that (DNR) is general enough to apply to any given field of inquiry (although, given the appeal to knowledge, normative fields such as ethics may be difficult cases). But, in contrast, (DNR) does not provide a clear strategy for deriving more substantive accounts of fruitfulness for specific fields of inquiry. For any given explication, according to (DNR), one is simply to look for the production of knowledge. There is nothing we can plug into (DNR) to obtain a more substantive, detailed account; there is no obvious way to recover the details we saw in the case of GENE above.

None of the concerns raised here are decisive. But they are suggestive. And, in what follows, I build on them to develop the Relevant-Goals Account of Fruitfulness.

\section{The Relevant-Goals Account of Fruitfulness}

I state the account in Sect. 3.1, develop it in Sects. 3.2 and 3.3, and give an example in Sect. 3.4.

\subsection{The Account}

Let me begin with a bald statement of the account.

\section{The Relevant-Goals Account of Fruitfulness.}

- An explicatum is fruitful insofar as its replacement of the corresponding explicandum would facilitate, through the ordinary course of inquiry, progress towards achieving relevant theoretical goals.

- Given multiple fruitful candidate explicata for a single explicatum, the most fruitful candidate is that whose replacement of the explicandum would facilitate, through the ordinary course of inquiry, most progress towards achieving relevant theoretical goals.

On this proposal, the theoretical goals that are relevant to a particular explication provide substantive criteria against which to measure fruitfulness when performing 
that particular explication. ${ }^{13}$ I will say more about how I am understanding theoretical goals in Sect. 3.2. But examples include: to formulate universal generalisations; to explain some particular phenomenon; or to develop a principled taxonomy of celestial objects. ${ }^{14}$

Notice that relevant theoretical goals are analogous to Kitcher's significant questions. I prefer to talk of goals rather than questions because, in some cases, it is difficult to formulate appropriate questions. In the planet case, theorists are plausibly guided in part by the theoretical goal to develop a principled taxonomy of celestial objects. Yet it is unclear how to reformulate this goal as a question. Perhaps: Is there a principled taxonomy of celestial objects?; or What are the details of a principled taxonomy of celestial objects?. But answering the former question need not involve developing a principled taxonomy of celestial objects; and answering the latter seems, if anything, to go beyond it. In contrast, questions are easily translated into goals: given a question, $Q$, we can formulate a corresponding goal, to answer $Q$. Thus, overall, I prefer to talk of relevant theoretical goals.

There are two principal ways that such progress can be facilitated towards achieving theoretical goals. Firstly, progress is facilitated if the goal is achieved by the very act of replacing the explicandum with the explicatum: the goal to provide a principled extension for "planet" was achieved by the very act of replacing PLANET ${ }_{\text {OLD }}$ with PLANET ${ }_{\text {NEW }}$. Secondly, progress is facilitated if a step is made towards achieving the goal by the act of replacing the explicandum with the explicatum: the goal to formulate empirical laws is at best brought a step closer by performing that replacement. There is no requirement that those performing the explication know that such a step has been successfully made so long as, in typical cases, theorists have a good idea of what counts as a move in the right direction. To say that progress is facilitated through the ordinary course of inquiry is to rule out atypical cases in which an explication results in progress towards achieving relevant theoretical goals in deviant ways - cases, for example, in which a 'bad' explication nonetheless inspires a theorist to look at a problem in a new and illuminating way.

When deciding between multiple fruitful candidate explicata, one compares how much progress would result from replacing the explicandum with each candidate. There is no general algorithm for such comparisons-progress is typically qualitative rather than quantitative - and relative fruitfulness must be assessed, using academic judgement, on a case-by-case basis. ${ }^{15}$ Moreover, different theorists, even those who share theoretical goals, may make different judgements on a given case. It does not automatically follow that one of the theorists is mistaken; there may always

\footnotetext{
13 Thus, one can effectively obtain each of Carnap's, Kitcher's and Dutilh Novaes and Reck's views as special cases. This is achieved by considering cases in which the relevant theoretical goals are, respectively: to formulate universal statements; to answer significant questions; and to produce new knowledge about the phenomena to which the explicatum pertains. Thanks to an anonymous referee for encouraging me to make this explicit.

14 Here and below: a taxonomy or extension is principled insofar as it coheres with contemporary understanding of the relevant phenomena.

15 Cf. Kuhn (1977) on theory choice.
} 
be a subjective element to such judgements. Nonetheless, in many cases, the judgements will be clear-cut. See Sect. 3.4 for an illustration of this process.

Finally, note that I make the simplifying assumption throughout that, in any given explication, relevant theoretical goals are to be weighted equally. Ultimately, we might drop the assumption. Suppose that, in judging candidate explicata for PLAN$\mathrm{ET}_{\mathrm{OLD}}$, we took the most significant factor to be whether candidates provided a principled division amongst celestial objects. This would be compatible with thinking that-all else being equal-a candidate explicatum that facilitated the formulation of empirical laws would have been better than one that did not. In such a case, there would have been (at least) two relevant theoretical goals-to develop a principled taxonomy of celestial objects and to formulate empirical laws-with the former weighted more heavily than the latter. However, the introduction of different weightings complicates the discussion of the account, without making the underlying ideas any more perspicuous. So, for present purposes, I put weighting aside.

\subsection{Theoretical Goals}

What, in the relevant sense, are theoretical goals? As a first gloss, one might be tempted to characterise theoretical goals simply as goals pertaining to some or other theoretical matter. However, herein, I have a thicker notion in mind. When performing explications, theorists should not aim simply to achieve goals that pertain to some or other theoretical matter. In addition, theorists should aim to thereby make appropriate progress. Now, what constitutes progress in inquiry plausibly varies between different fields: progress in ethics may look nothing like progress in astronomy. To keep things manageable, I will focus on theoretical goals in science. That is, in this subsection, I will sketch a view of theoretical goals in the specific sense of: goals the achievement of which would lead to progress in scientific inquiry. ${ }^{16}$

I have already given several examples of what I am taking to be theoretical goals: to explain some phenomenon; to formulate universal generalisations; to formulate empirical laws; to develop a taxonomy of celestial objects; to provide a principled extension for "planet". A notable feature of such goals is that, if achieved, they might genuinely lead to better scientific theories: if phenomenon $P$ falls within the scope of some scientific theory, then that theory is ceteris paribus better if it explains $P$; all else being equal, a scientific theory that includes (true) universal generalisations or empirical laws is better than one that does not; and our best astronomical theories are overall improved by the development of a principled taxonomy of celestial objects and a principled extension for "planet". I recommend, then, that we understand theoretical goals in terms of theoretical values.

Theoretical values are general characteristics of good scientific theories, such as: internal consistency; coherence (with other accepted theories); evidential accuracy (i.e. fit with the evidence); scope (i.e. applicability to a wide range of phenomena and/or cases); explanatory power; simplicity; and so on. Roughly, the more

16 Thanks to an anonymous referee for pushing me to clarify this. 
theoretical values a theory has, and the greater extent to which it has them, the better the theory. But there are complications: some values might be weighted more heavily than others (such as internal consistency over simplicity), and values might sometimes conflict (increasing scope can decrease evidential accuracy). There are also important questions about what the theoretical values in fact are, how they are related, and why they are valued. For present purposes, however, I put such complications and questions aside. ${ }^{17}$

We can give an account of theoretical goals in terms of theoretical values: a goal pertaining to some scientific theory is theoretical insofar as achievement of that goal would ipso facto enhance some theoretical value(s) of that theory. Call this view Value-Directedness. More precisely, where $T$ is a scientific theory:

A goal is theoretical with respect to $T$ insofar as: ceteris paribus, achievement of that goal would ipso facto transform $T$ into a theory that is better with respect to some theoretical value(s) than $T$.

For example, the formulation of new universal generalisations and empirical laws may ipso facto increase the scope of a theory. That is, whereas $T$ may only apply to individual cases the transformed theory will apply across the board; so, other things being equal, the transformed theory will have greater scope than $T$. According to Value-Directedness, then, to formulate universal generalisations and to formulate empirical laws will in such cases count as theoretical goals.

Similarly for the goal to explain phenomenon $P$. If $\mathrm{P}$ falls within the scope of a theory that does not explain it, then providing that theory with the resources to explain P may ipso facto enhance its explanatory power. That is, whereas $T$ might not explain $\mathrm{P}$, the transformed theory will do; so, other things being equal, the transformed theory will have greater explanatory power than $T$. According to ValueDirectedness, then, to explain phenomenon $P$ will in such cases count as theoretical goals.

Finally, consider the goals to develop a principled taxonomy of celestial objects and to provide a principled extension for "planet". The concept PLANET ${ }_{\mathrm{OLD}}$ was not principled, in the sense that it did not cohere well with contemporary understanding of the solar system: given the discovery of Eris, contemporary understanding suggested that PLANET ${ }_{\text {OLD }}$ did not denote an astronomically important category. So, one effect of developing a principled taxonomy of celestial objects, and of providing a principled extension for "planet", was that the planetary sciences would henceforth cohere better with astronomical theories more generally; a theory of planetary formation, say, would henceforth cohere better with a theory of the evolution of solar systems. Thus, achievement of those goals would ipso facto increase the coherence of a variety of astronomical theories. So according to Value-Directedness, for the IAU, to develop a principled taxonomy of celestial objects and to provide a principled extension for "planet" counted as theoretical goals.

\footnotetext{
17 The classic exposition of theoretical values is Kuhn (1977). For development and discussion, see e.g.: Laudan (2004), Douglas (2013), McMullin (2014) and Keas (2018).
} 
Implicit in the above comments is the thought that goals do not count as theoretical simpliciter, but only with respect to some scientific theory. Providing a principled extension for "planet" may enhance the scope of a theory of planetary science, but is unlikely to enhance any theoretical values of a theory of (say) genetics. Generalising, we can also say that a goal is theoretical with respect to a whole scientific field. Successfully unifying quantum and relativistic physics would ipso facto transform theories in both fields, such that the new theories would by-and-large be better with respect to (say) coherence than the old theories. The goal to unify quantum and relativistic physics, then, is theoretical with respect to the fields of quantum and relativistic physics.

As it stands, Value-Directedness may not generalise to non-scientific fields of inquiry. Several questions are relevant here. What exactly are scientific theories? To what extent are they like the theories we find in other fields of inquiry? Are theoretical values applicable to non-scientific theories and, if so, to what extent? Does progress in other disciplines consist of developing better theories? If we think of philosophy as broadly continuous with science, then we might think that the traditional theoretical values apply mutatis mutandis in philosophy. ${ }^{18}$ But, in contrast, if we focus instead on the normative nature of philosophical inquiry, then many of the traditional theoretical values seem inappropriate. It is unclear that we can straightforwardly measure progress in ethics with reference to evidential accuracy or explanatory power, for example. These are deep issues about the nature of scientific and other inquiry, and I cannot resolve them here. For present purposes, I settle for Value-Directedness, construed only as an account of theoretical goals in scientific inquiry.

\subsection{Relevance}

The Relevant-Goals Account ties fruitfulness to relevant theoretical goals. So which theoretical goals are relevant? I offer an answer to this question, Contextualism, in this subsection. ${ }^{19}$

Explications are performed for particular theoretical purposes. So, given that fruitfulness is the principal desideratum for explicata, it makes good sense to measure fruitfulness against those purposes. Contextualism is intended to capture this thought by tying relevant theoretical goals to whoever or whatever is performing a given explication. More precisely, Contextualism is the view that, for any given explication, the relevant theoretical goals are the explicator's theoretical goals. How this is to be understood depends on what sort of entity - say, theorist or institutionis performing the explication.

\footnotetext{
18 See e.g. Ladyman (2012).

19 An alternative view is that the relevant theoretical goals are the objective aims of the corresponding field of inquiry - be it science, philosophy, or something else. As indicated in Sect. 2, however, I prefer to avoid building such objective aims into our account of fruitfulness.
} 
First, suppose that an individual theorist is performing an explication. Then: the explicator's theoretical goals are the personal theoretical goals of that individual theorist qua performer of the explication. This requires explanation.

Let personal theoretical goals be an individual theorists' theoretical goals. For example, some astronomers may have a theoretical goal to explain the properties of Pluto; some may have a theoretical goal to provide a principled taxonomy of transNeptunian objects; some may have a theoretical goal to develop universal generalisations about different varieties of celestial objects; and so on. ${ }^{20}$ An individual theorist may have many different theoretical goals, and these goals may change over time.

Now, theorists often seek piecemeal progress, tackling only some of their personal theoretical goals at any given time. Accordingly, we can distinguish between the personal theoretical goals of a theorist as such, and those of a theorist qua performer of an explication. The personal theoretical goals of a theorist as such are standing theoretical goals towards which, in general, she is working; the personal theoretical goals of a theorist qua performer of an explication are those theoretical goals towards which, in performing that explication, she is working. For example, suppose that Brown sought singlehandedly to explicate PLANET $_{\mathrm{OLD}}$. She might have had various standing personal theoretical goals-such as, say, to provide a principled taxonomy of trans-Neptunian objects and to predict and explain the conditions at the very edge of the solar system. These were Brown's personal theoretical goals as such. However, in relation to the explication of PLANET ${ }_{\text {OLD }}$, Brown may have had a more focused set of theoretical goals - say, to provide a principled taxonomy of trans-Neptunian objects and, in particular, to determine which, if any, trans-Neptunian objects are to fall under "planet", with no reference at all to the conditions at the edge of the solar system. In this case, then, the explicator's theoretical goalsand thus, according to Contextualism, the relevant theoretical goals-would be: to provide a principled taxonomy of trans-Neptunian objects; and to determine which, if any, trans-Neptunian objects are to fall under "planet".

Second, suppose that an institution is performing the explication. Then: the explicator's theoretical goals are the institutional theoretical goals of that institution qua performer of the explication. Here, let institutional theoretical goals be theoretical goals that are (explicitly) endorsed by research groups, centres or institutions. For example, the Centre for the Study of the Senses, which is hosted by the Institute of Philosophy at the School of Advanced Study at the University of London, states that its

overall aim is to achieve a coherent framework to accommodate the welter of recent findings that have revised our understanding of how the different senses contribute to our perception of the environment, and awareness of ourselves. ${ }^{21}$

\footnotetext{
${ }^{20}$ In this subsection, I generally omit the defence of claims that such-and-such goals are theoretical.

21 From http://philosophy.sas.ac.uk/centres/censes. Accessed 6th March 2019.
} 
This goal is theoretical given that, ceteris paribus, achieving it would enhance the coherence of the plurality of theories that we might collectively label contemporary sense sciences.

Just like individuals, institutions often seek piecemeal progress, tackling only some of their institutional theoretical goals at any given time. Accordingly, we can distinguish between the theoretical goals of an institution as such, and those of an institution qua performer of an explication. For example, the Centre for the Study of the Senses has standing institutional theoretical goals as quoted above. But, on a given occasion, the Centre might seek in particular to provide a set of clearlydefined taxa of interoceptive senses (e.g. 'pain', 'hunger', 'itchiness') that reflect current understanding without reference to how our senses contribute to our perception of the environment. In such a case, the explicator's theoretical goals - and thus, according to Contextualism, the relevant theoretical goals-would be (say): to achieve a coherent framework to accommodate the recent findings that have revised our understanding of our awareness of ourselves; and to provide a set of clearlydefined taxa of interoceptive senses (e.g. 'pain', 'hunger', 'itchiness') that reflect current understanding.

On this view, explicators have a significant degree of control over which of their theoretical goals are relevant, and thus over the measure of fruitfulness that applies in a given case. This does not imply that anything goes. Firstly, as relevant theoretical goals are always theoretical goals, they must (by Value-Directedness) be directed towards the improvement of certain theories in certain respects. An explicator cannot simply decide to uniformly degrade theories in the name of fruitfulness. Secondly, additional normative factors, external to the method of explication, may constrain explicators' theoretical goals. For example, many contemporary philosophers would judge the following theoretical goal to be a dead-end: to reduce all scientific statements to statements about sense-data. If that is right then, on grounds independent to the method of explication, explicators ought not to have that goal. So, faced with a contemporary theorist who (say) seeks to explicate OBJECT as a first step to reducing all scientific statements to statements about sense-data, we might say two things: that, given her specific context, there may be fruitful explicata available; but that, nonetheless, there are good reasons not to be in that context.

That, then, is Contextualism-the view that the theoretical goals relevant to an explication are the explicator's theoretical goals. A few brief comments in its favour are in order. First, as noted above, Contextualism is a natural view given that explications are performed for specific theoretical purposes. Given Contextualism, those specific theoretical purposes are modelled by the relevant theoretical goals. Second, Contextualism provides a recipe for deriving clear, substantive and detailed accounts of fruitfulness for specific explications. The Relevant-Goals Account is thus general enough to apply to any given explication. And, by providing additional details about the explicator's theoretical goals, we can derive a substantive measure for fruitfulness in that case. This parallels the twofold benefit of Kitcher's account, discussed in Sect. 2.

Third, given Contextualism, we have good reason to think that the Relevant-Goals Account can be practically helpful for those performing explications. Theoretical goals are the sort of thing that can guide an explication, but only if those performing 
the explication have straightforward epistemic access to which theoretical goals are their guides. And given Contextualism, those performing an explication do have such epistemic access, because they have epistemic access to their theoretical goals. In large part, this is simply because those performing explications-whether individual theorists or institutions-determine what their theoretical goals are in each case. Given Contextualism, the Relevant-Goals Account provides practical guidance for the performance of explications.

\subsection{Example}

The IAU considered two candidate explicata when explicating PLANET $_{\text {OLD }}$, PLANET $_{\text {DRAFT }}$ and PLANET $_{\mathrm{NEW}}$, ultimately choosing PLANET $_{\mathrm{NEW}}$. I now argue that, on the present picture, this was the right choice.

The IAU states several aims on its website, at least of which two are theoretical goals: to provide "unambiguous [i.e. clearly defined] astronomical nomenclature" (achieving which would ceteris paribus ipso facto enhance the scope of astronomical theories); and to define "fundamental astronomical and physical constants" (achieving which would ceteris paribus ipso facto enhance the evidential accuracy of astronomical theories). ${ }^{22}$ These are the IAU's institutional theoretical goals as such. In explicating PLANET ${ }_{\text {OLD }}$, however, the IAU was working only towards the former. So, of these, only

\section{(I) to provide clearly-defined astronomical nomenclature}

was a relevant theoretical goal in the planet case.

The IAU also had a more specific aim. In the resolution documenting the explication, the IAU writes that:

[...] it is important that our nomenclature for objects [in planetary systems] reflect our current understanding. This applies, in particular, to the designation "planets". [...] Recent discoveries lead us to create a new definition, which we can make using currently available scientific information. (2006b: 1)

More succinctly and explicitly, the IAU is here endorsing the following goal:

(II) to provide a taxonomy for celestial objects that reflects our current understanding (with specific reference to "planet").

This goal is theoretical: achieving it would ipso facto enhance the coherence of the planetary sciences. As such, it was a relevant theoretical goal in the planet case.

Accepting that (I) and (II) are the only relevant theoretical goals, in order to determine which candidate promised to be most fruitful we must consider the extent

\footnotetext{
22 From https://www.iau.org/about/. Accessed 6th March 2019. Other aims include: to organise scientific meetings; to promote educational activities; and to discuss the possibility of "future international largescale facilities".
} 
to which replacing PLANET ${ }_{\mathrm{OLD}}$ with PLANET $_{\text {DRAFT }}$ or PLANET $_{\mathrm{NEW}}$ would have facilitated progress towards achieving (I) and (II).

First, consider (I). PLANET ${ }_{\text {OLD }}$ did not serve to underpin clearly-defined nomenclature: as the concept is defined only by heterogeneous instances and non-instances, it fails to classify many celestial objects in our solar system and provides no principled basis for extension to objects in other solar systems. In virtue of their explicit definitions, in contrast, both PLANET DRAFT $_{\text {and }}$ PLANET ${ }_{\text {NEW }}$ were well-placed both to classify the celestial objects in our solar system and to be extended to objects in other solar systems. Neither candidate underpins a perfectly clear nomenclature. There are some difficulties spelling out: how spherical an object must be to satisfy condition (b) of both definitions; and to what extent an object must have cleared its object of debris to satisfy condition (c) of the definition of PLANET $_{\mathrm{NEW}}{ }^{23}$ Either of these difficulties could, in principle, have led to unclarity in nomenclature as more celestial objects were discovered, but there is no a priori reason to think that either candidate explicatum would be more likely than the other to lead to such problems. So, by replacing PLANET ${ }_{\text {OLD }}$ with either candidate explicatum, the IAU would have taken a clear step towards achieving (I); and the IAU had no reason to predict that either replacement would have constituted a greater step. From the IAU's perspective, then: with respect to (I), both PLANET ${ }_{\text {DRAFT }}$ and PLANET $_{\text {NEW }}$ were fruitful, with neither being clearly more fruitful than the other.

Second, consider (II). In light of the discovery of Eris and other trans-Neptunian objects very similar to Pluto, it was clear that PLANET ${ }_{\text {OLD }}$ did not reflect contemporary understanding of the solar system. As both PLANET ${ }_{\text {DRAFT }}$ and PLANET $_{\text {NEW }}$ were defined in terms of well-understood celestial properties, replacing PLANET $_{\mathrm{OLD}}$ with either candidate explicatum would have constituted a step towards achieving (II). That is, with respect to (II), both PLANET ${ }_{\text {DRAFT }}$ and PLANET ${ }_{\text {NEW }}$ were fruitful.

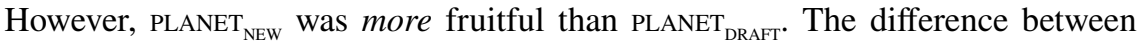
the two concepts is that PLANET ${ }_{\mathrm{NEW}}$ contains an additional clause: to fall under PLANET $_{\mathrm{NEW}}$, a celestial object $x$ must be such that

(c) $x$ has cleared its neighbourhood of debris.

Now, while no celestial object in our solar system has cleared its neighbourhood of debris completely, it was known that there is significant difference between the extent to which Mercury through to Neptune have cleared their neighbourhoods of debris, and the extent to which other celestial objects have cleared their neighbourhoods of debris. ${ }^{24}$ Following Soter (2006), let $\mu$ be the ratio of the mass of a celestial object and the total combined mass of other objects in its neighbourhood. Then the value of $\mu$ for each of Mercury through to Neptune is over $5 \times 10^{3}$. By comparison,

\footnotetext{
23 See Soter (2006).

24 See e.g. Soter (2006) and Stern and Levison (2002). Note that (c) may ultimately need to be adjusted to reflect the fact that no celestial object in our solar system has cleared its neighbourhood of debris completely.
} 
the value for Pluto and Eris is 0.07 and 0.1 respectively; and the value for the largest asteroid in the solar system is 0.33 .

Importantly, in a sufficiently mature solar system (such as our own), these differences in $\mu$-value track a key distinction in our understanding of how that solar system evolved. As a solar system evolves, dust and gas orbiting a star will be collected up into bodies. At any given distance from the sun, a single dominant body may emerge that scatters or subsumes remaining debris in its neighbourhood. In a mature solar system, an object with a high $\mu$-value is a dominant body that has reached the end of this process; an object with a low $\mu$-value, however, is effectively "stranded in [an] arrested stage of development": the dynamics of the solar system in its mature state prevent such an object from becoming dominant in its neighbourhood (Soter 2006: 2517).

The astronomical details need not concern us here. But, if this is right, then PLANET $_{\mathrm{NEW}}$ reflected an important aspect of contemporary understanding of celestial objects that was not reflected by PLANET $_{\text {DRAFT }}$. Replacing PLANET $_{\mathrm{OLD}}$ with PLANET $_{\mathrm{NEW}}$, rather than PLANET ${ }_{\mathrm{DRAFT}}$, would thus have been the greater step towards providing a taxonomy for celestial objects that reflected contemporary understanding. That is, with respect to (II), PLANET $_{\mathrm{NEW}}$ was more fruitful than PLANET $_{\mathrm{DRAFT}}$.

Overall, then, while both PLANET ${ }_{\mathrm{NEW}}$ and PLANET $\mathrm{DRAFT}$ were fruitful, PLANET ${ }_{\mathrm{NEW}}$ was more fruitful than PLANET ${ }_{\text {DRAFT. }}$ Thus, given these two candidate explicata, the IAU were right to replace PLANET ${ }_{\mathrm{OLD}}$ with PLANET $_{\mathrm{NEW}}$.

\section{Dynamics}

A possible concern about the foregoing is that it presupposes an implausible 'static' model of inquiry, as if inquiry consists of isolated activities aimed at achieving fixed goals. I develop the concern, in Sect. 4.1, as two separate objections. I respond to both objections, in Sect. 4.2, by explaining how the Relevant-Goals Account fits into the 'dynamic' nature of inquiry.

\subsection{Two Objections}

The first objection is that the Relevant-Goals Account represents theorists (and institutions) as having fixed theoretical goals towards which they are working. However, in reality, inquiry involves the ongoing revision and refining of one's theoretical goals.

For example, in the planet case, the relevant theoretical goal (II), to provide a taxonomy for celestial objects that reflects our current understanding (with specific reference to "planet"), was only explicitly formulated at the point of explication. The goal, however, was not plucked out of thin air; it was the result of the changing contexts through which the controversy about the status of Pluto unfolded. ${ }^{25}$ Upon

\footnotetext{
${ }^{25}$ See e.g. Tyson (2009).
} 
discovery of Eris, the principal goal was perhaps just to determine whether Eris is a planet. But, given the similarity of Eris to Pluto, this evolved into the goal to determine whether Pluto is a planet. To bypass the issue, the Hayden Planetarium (in 2000) changed its displays to use only the relatively uncontroversial designations "Terrestrial Planet" (Mercury through to Mars) and "Jovian Planet" (Jupiter through to Neptune), without reference to Pluto at all. Media attention turned this into a public controversy, which led theorists to seek to determine whether "planet" denotes an astronomically useful category. In part due to social pressures supporting the designation "planet", the principal goal evolved further into the goal to provide a definition of "planet" that reflects current understanding. It is in this context that the IAU voted for PLANET ${ }_{\mathrm{NEW}}$.

This 'history' of the theoretical goal (II) remains highly artificial, but is nonetheless sufficient to emphasise that theoretical goals are not fixed. The concern is that the Relevant-Goals Account misrepresents the way that theoretical goals change through time; and that this misrepresentation is sufficiently severe to undermine the account as a model of what theorists should aim for in engineering concepts.

The second objection is that the Relevant-Goals Account represents conceptual engineering as being isolated from other theoretical practice, as if theorists (and institutions) periodically pause their other activities to engineer a concept. However, the different activities that constitute an inquiry-reading, thinking, collecting and analysing data, formulating arguments, considering objections, discussing with colleagues, refining ideas, engineering concepts, etc.- - are in fact thoroughly intertwined, with the different activities combining to bring about theoretical progress. Conceptual engineering is thus embedded within the ongoing activities that constitute an inquiry.

This point can also be brought out by considering the planet case. As noted above, the IAU's definition (a)-(c) is not, as it stands, adequate: none of Mercury through to Neptune have completely cleared their orbits of debris. Soter's clarification of (c), discussed in Sect. 3.4, is in effect a further conceptual refinement, a further explication of the IAU's explicatum that yields ${ }^{26}$ :

A celestial object $x$ falls under PLANET SOTER $_{\text {iff: }}$

(a) $x$ orbits the sun;

(b) $x$ is sufficiently large for its own gravity to have formed it into a sphere; and

(c) the value of $\mu$ for $x$ is high (say, $\left.\mu_{x}>100\right){ }^{27}$

It was in performing this further explication that Soter explicitly tied the designation "planet" to the evolution of the solar system, highlighting a clear theoretical basis for the definition. Now, even in this simple case, the changing definition of "planet" is best seen as embedded within broader theoretical work: we cannot clearly separate Soter's refinement of the definition of "planet" from the simultaneous development

\footnotetext{
26 See Dutilh Novaes and Reck (2017: 201f) for discussion of the iterative nature of explication, the fact that "the explicatum of an explication may become the explicandum for another explication".

27 Soter (2006: 2517) also recommends dropping (b), although for simplicity I leave this point aside here.
} 
of the theory of the evolution of the solar system. The theoretical development is at once the precursor to, and consequence of, the redefinition; a justification for, and justified by, the redefinition. More generally, the ongoing engineering of concepts is embedded within the ongoing development of theories, ideas, data, and so on.

The underlying concern, then, is the same as before-that the Relevant-Goals Account misrepresents actual theoretical practice, undermining the account as a model of what theorists should aim for in engineering concepts.

\subsection{Dynamics and the Method of Explication}

As noted at the outset, I see the method of explication as normative: it is a recommendation for how to go about engineering concepts in inquiry, rather than a description of how theorists in fact go about engineering concepts. For this reason, the apparent disconnect between the Relevant-Goals Account and the dynamic nature of theoretical practice is only a problem if the disconnect prevents the account from being a plausible recommendation.

Now, for the method of explication to be of practical value, it must simplify to some extent. A method that explicitly takes into account all of the complexities of inquiry would itself be too complex to provide helpful guidance. A simpler method, an idealisation that can be adapted on the fly to the situation at hand, is likely to be preferable. By analogy, speaking to a new driver in the UK, one might recommend that she drive on the left-hand side of the road. The driver can then adapt this advice as need be-for example, when navigating narrow lanes on which she is forced to drive in the middle of the road, when driving past parked cars which require her to move over to the right-hand side, when driving down multiple-lane one-way streets at the end of which she wants to turn right, and so on. It would not be helpful, so much as overwhelming, to try to take all of these complexities into account when offering the initial recommendation to the driver. When recommending a strategy for navigating something complex, simplifications are invaluable.

Plausibly, then, the normative nature of the method of explication justifies a certain degree of simplification. But, according to the objections, the Relevant-Goals Account does not so much simplify as misrepresent: the Relevant-Goals Account misrepresents a dynamic process as static. If this is right, then the above line of response may seem unconvincing. However, contrary to the objections, the Relevant-Goals Account does not misrepresent inquiry. Although the account does not make the dynamic nature of inquiry explicit, it does not imply that inquiry is, in the relevant sense, static. Let me explain.

First, consider the fact that a theorist's theoretical goals can change. Contrary to the first objection, the Relevant-Goals Account does not in fact represent such theoretical goals as fixed; the account says nothing about whether they change or not. According to the account, at any given moment, the theoretical goals that are driving an explication also serve as the measure of fruitfulness for that explication. If the theoretical goals change, then so does the measure. The Relevant-Goals Account is thus in principle compatible with the fact that a theorist's theoretical goals can change. 
Second, consider the fact that concepts are not engineered in isolation from a theorist's other theoretical practice. Contrary to the second objection, the RelevantGoals Account does not in fact represent conceptual engineering as isolated in this way; the account says nothing about the context in which conceptual engineering takes place. There may be several other activities going on in concert. The RelevantGoals Account says only that, whatever those activities are, we measure the fruitfulness of an explicatum against the theoretical goals that are driving the explication. The account is in principle compatible with the fact that conceptual engineering is embedded within a wide range of other theoretical activities.

So let me say how the Relevant-Goals Account is to be interpreted in practice. When a theorist is performing an explication, she should begin by formulating relevant theoretical goals. What is she trying to achieve? What is the intended theoretical advance? Her answers to these questions will be heavily informed by whatever other relevant theoretical activities she is engaged in. A string theorist trying to prove a theorem will have quite different aims from the astronomer trying to develop a principled taxonomy of celestial objects. The theorist can proceed by sketching some possible definitions for candidate explicata. These may undergo preliminary assessment: the more fruitful, the better. It is unlikely that the theorist will be immediately satisfied with her definitions: conceptual engineering is hard. However, several options are open to the unsatisfied theorist. Firstly, she might seek to tighten up and improve the definitions for her candidate explicata. Secondly, she might try a different strategy, seeking brand new candidate explicata. Thirdly, she might adjust her theoretical goals in light of what she has learnt. (Perhaps, for example, the string theorist realises that the claim she is trying to prove needs to be weakened in some way.) Fourthly, she might go back to her other theoretical activities. (Perhaps, for example, the string theorist turns back to developing the proof, if only to see just what does follow from her definition.) Whatever the details, the theorist continues to adjust her theoretical goals, engage in other theoretical activities, and refine her candidate explicata until she is satisfied. When she is satisfied, and has settled upon an explicatum, then she counts as having performed an explication.

Note that there is no precise moment in this story at which the theorist performs the explication. And there is no guarantee that the end result speaks to the theoretical goals she had at the outset. But this merely reflects that her conceptual engineering efforts are intertwined with her other activities, all of which are in a feedback loop with her theoretical goals. What the Relevant-Goals Account specifically recommends is that the theorist keep her most up-to-date theoretical goals in mind throughout the process. Formulate those goals, refine them, and work towards achieving them.

Rather than misrepresenting inquiry as static, then, the Relevant-Goals Account de-emphasises its dynamic nature in order to provide a recommendation that can be adapted on the fly, as need be. The recommendation is that, when engineering a 
concept in inquiry, the aim be to make progress towards achieving the explicator's theoretical goals. $^{28}$

Open Access This article is licensed under a Creative Commons Attribution 4.0 International License, which permits use, sharing, adaptation, distribution and reproduction in any medium or format, as long as you give appropriate credit to the original author(s) and the source, provide a link to the Creative Commons licence, and indicate if changes were made. The images or other third party material in this article are included in the article's Creative Commons licence, unless indicated otherwise in a credit line to the material. If material is not included in the article's Creative Commons licence and your intended use is not permitted by statutory regulation or exceeds the permitted use, you will need to obtain permission directly from the copyright holder. To view a copy of this licence, visit http://creativecommons.org/licen ses/by/4.0/.

\section{References}

Bielik, L. (2018). Explication, H-D confirmation, and simplicity. Erkenntnis, 83(5), 1085-1104.

Brun, G. (2016). Explication as a method of conceptual re-engineering. Erkenntnis, 81(6), 1211-1241.

Brun, G. (2017). Conceptual re-engineering: from explication to reflective equilibrium. Synthese. https://doi. org/10.1007/s11229-017-1596-4.

Carnap, R. (1947). Meaning and necessity. Chicago: University of Chicago Press.

Carnap, R. (1962). Logical foundations of probability (2nd ed.). Chicago: The University of Chicago Press.

Carnap, R. (1963). Replies and systematic expositions. In P. Schilpp (Ed.), The philosophy of Rudolf Carnap (pp. 859-1013). Lasalle, IL: Open Court.

Carnap, R. (1966). Philosophical foundations of physics. London: Basic Books.

Carus, A. W. (2007). Carnap and twentieth-century thought: Explication as enlightenment. Cambridge: Cambridge University Press.

Douglas, H. (2013). The value of cognitive values. Philosophy of Science, 80(5), 796-806.

Dutilh Novaes, C., \& Reck, E. (2017). Carnapian explication, formalisms as cognitive tools, and the paradox of adequate formalization. Synthese, 194(1), 195-215.

International Astronomical Union. (2006a). The IAU draft definition of "planet" and "plutons" (Press Release iau0601). Retrieved July 7, 2017, from https://www.iau.org/news/pressreleases/detail/iau0601/.

International Astronomical Union. (2006b). Resolution B5: Definition of a planet in the solar system. Retrieved April 4, 2017, from https://www.iau.org/static/resolutions/Resolution_GA26-5-6.pdf.

Justus, J. (2012). Carnap on concept determination: Methodology for philosophy of science. European Journal for Philosophy of Science, 2(2), 161-179.

Keas, M. (2018). Systematizing the theoretical values. Synthese, 195(6), 2761-2793.

Kitcher, P. (2008). Carnap and the caterpillar. Philosophical Topics, 36(1), 111-127.

Kuhn, T. (1977). Objectivity, value judgement, and theory choice. In T. Kuhn (Ed.), The essential tension (pp. 320-339). Chicago: Chicago University Press.

Ladyman, J. (2012). Science, metaphysics and method. Philosophical Studies, 160(1), 31-51.

Laudan, L. (2004). The epistemic, the cognitive, and the social. In P. Machamer \& G. Wolters (Eds.), Science, values, and objectivity (pp. 14-23). Pittsburgh: University of Pittsburgh Press.

Maher, P. (2007). Explication defended. Studia Logica, 86(2), 331-341.

McMullin, E. (2014). The virtues of a good theory. In M. Curd \& S. Psillos (Eds.), The Routledge companion to philosophy of science (pp. 561-571). New York: Routledge.

Pinder, M. (2017a). Does experimental philosophy have a role to play in Carnapian explication? Ratio, 30(4), 443-461.

Pinder, M. (2017b). On Strawson's critique of explication as a method in philosophy. Synthese. https://doi. org/10.1007/s11229-017-1614-6.

Pinder, M. (2019). Conceptual engineering, metasemantic externalism and speaker-meaning. Mind. https:// doi.org/10.1093/mind/fzz069.

\footnotetext{
${ }^{28}$ For helpful comments, I would like to thank several anonymous referees, as well as audiences in Birmingham, Bristol, Oslo and Southampton.
} 
Quine, W. V. O. (1960). Word and object. Cambridge, MA: The MIT Press.

Rowbottom, D. (2014). Aimless science. Synthese, 191(6), 1211-1221.

Schupbach, J. (2017). Experimental explication. Philosophy and Phenomenological Research, 94(3), 672-710.

Shepherd, J., \& Justus, J. (2015). X-phi and Carnapian explication. Erkenntnis, 80(2), 381-402.

Soter, S. (2006). What is a planet? Astronomical Journal, 132(6), 2513-2519.

Stern, S. A., \& Levison, H. (2002). Regarding the criteria for planethood and proposed planetary classification schemes. Highlights of Astronomy, 12, 205-213.

Strawson, P. F. (1963). Carnap's views on constructed systems versus natural languages in analytic philosophy. In P. Schilpp (Ed.), The philosophy of Rudolf Carnap (pp. 503-518). Lasalle IL: Open Court.

Tyson, N. (2009). The Pluto files: The rise and fall of America's favorite planet. New York: Norton and Co.

Publisher's Note Springer Nature remains neutral with regard to jurisdictional claims in published maps and institutional affiliations. 